\title{
FORMATION OF SINGULARITIES IN COMPRESSIBLE FLUIDS IN TWO-SPACE DIMENSIONS
}

\author{
M. A. RAMMAHA
}

(Communicated by Barbara Lee Keyfitz)

\begin{abstract}
Classical solutions to the two-dimensional Euler equations for a polytropic ideal fluid are considered. It is shown that any local $C^{1}$-flow, regardless of the size of the initial disturbance, will develop singularities in finite-time provided the front of the initial disturbance satisfies certain conditions.
\end{abstract}

\section{INTRODUCTION}

In this article we consider the motion of a polytropic ideal fluid in two-space dimensions. Such motion is described by the compressible Euler equations

$$
\begin{aligned}
\rho_{t}+\nabla \cdot(\rho u) & =0 \\
\rho\left(u_{t}+(u \cdot \nabla) u\right)+\nabla p & =0 \\
S_{t}+u \cdot \nabla S & =0 \\
p=p(\rho, S)=A \rho^{\gamma} e^{S}, \quad(A & >0, \gamma>1) .
\end{aligned}
$$

Here, $\rho$ is the density, $u=\left(u_{1}, u_{2}\right)$ is the velocity, $S$ is the specific entropy, and $p$ is pressure of the fluid. In the equation of state (1.4), $\gamma$ is the adiabatic index and $A$ is a positive constant.

The Cauchy data are

$$
\rho(x, 0)=\rho^{0}(x)>0, \quad u(x, 0)=u^{0}(x), \quad S(x, 0)=S^{0}(x) .
$$

The following assumption will be valid throughout the paper: there exists $R>0$ such that

$$
\rho^{0}(x)=\rho_{0}>0, \quad u^{0}(x)=0, \quad S^{0}(x)=S_{0},
$$

for $|x| \geqq R . \rho_{0}$ and $S_{0}$ are constants.

Equations (1.1)-(1.4) form a positive symmetric hyperbolic system in the variables $(\rho, u, S)$, one for which we can construct a unique local classical solution defined on some finite interval of time $[0, T)$, provided the Cauchy data are sufficiently regular [4].

Received by the editors August 30, 1988 and, in revised form, February 21, 1989.

1980 Mathematics Subject Classification (1985 Revision). Primary 35L45, 35L67.

This research was supported by the Office of Naval Research under Contract N00014-88-K-0376. 
The maximum speed of propagation of the front of a smooth disturbance is governed by the sound speed $\sigma$. Since $u^{0}(x)=0$ for $|x| \geqq R$, then

$$
\sigma=\left[\frac{\partial}{\partial \rho} p\left(\rho_{0}, S_{0}\right)\right]^{1 / 2}=\left[A \gamma \rho_{0}^{\gamma-1} e^{S_{0}}\right]^{1 / 2}
$$

The purpose of this paper is to show that any local $C^{1}$-solution $(\rho, u, S)$ of the equations (1.1)-(1.4), regardless of the size of the initial disturbance, will develop singularities in finite-time whenever the front of the initial disturbance satisfies certain positivity conditions. We, however, do not address in this article the question concerning the nature of such singularities. The mentioned positivity conditions are made precise in the statement of Theorem 2. They simply state that, on the average, the initial disturbance is slightly compressed and out-going in the $x_{1}$ or $x_{2}$ direction.

The following Lemma is a consequence of local energy estimates obtained by Sideris [9].

Lemma 1. Let $(\rho, u, S) \in C^{1}\left(\mathbf{R}^{2} \times[0, T)\right)$ be a solution of (1.1)-(1.4), (1.5a, b). Then $(\rho, u, S)=\left(\rho_{0}, 0, S_{0}\right)$ for all $|x| \geqq R+\sigma t, 0 \leqq t<T$.

Now, define the functions

$$
\begin{aligned}
m(t) & =\int_{\mathbf{R}^{2}}\left(\rho(x, t)-\rho_{0}\right) d x \\
\eta(t) & =\int_{\mathbf{R}^{2}}\left[\rho(x, t) \exp \left[\frac{1}{\gamma} S(x, t)\right]-\rho_{0} \exp \left[\frac{1}{\gamma} S_{0}\right]\right] d x,
\end{aligned}
$$

and

$$
F(t)=\int_{\mathbf{R}^{2}} \rho(x, t) x \cdot u(x, t) d x .
$$

As long as $(\rho, u, S)$ is smooth, it is then easy to show that $m(t)=m(0)$ and $\eta(t)=\eta(0)$. It is stated in Theorem 1 below that singularities in $C^{1}$ compressible flows in two-space dimensions are developed due to large initial disturbances. The proof of Theorem 1 is omitted since it is identical to the one given by Sideris [11] for compressible flows in three-space dimensions.

Theorem 1. Let $(\rho, u, S) \in C^{1}\left(\mathbf{R}^{2} \times[0, T)\right)$ be a solution of (1.1)-(1.4), (1.5a, b). If $m(0) \geqq 0, \eta(0) \geqq 0$, and $F(0) \geqq 3 \pi \sigma R^{3}\left\|\rho^{0}\right\|_{\infty}$, then $T$ is necessarily finite.

In order to state our main result, we define

$$
q^{0}(r)=\int_{x_{1}>r}\left(x_{1}-r\right)^{2}\left(\rho^{0}(x)-\rho_{0}\right) d x,
$$

and

$$
q^{1}(r)=\int_{x_{1}>r}\left(x_{1}-r\right) \rho^{0}(x) u_{1}^{0}(x) d x
$$


where $u_{1}^{0}$ is the first component of $u^{0}(x)=\left(u_{1}^{0}(x), u_{2}^{0}(x)\right)$. Note that, $q^{0}(r)=$ $q^{1}(r)=0$ for $r \geqq R$.

In what follows, all generic constants will be denoted by $c$; they may depend on the fixed constants $R$ and $R_{0}$, but otherwise are totally independent of the initial data.

Theorem 2. Let $(\rho, u, S) \in C^{1}\left(\mathbf{R}^{2} \times[0, T)\right)$ be the solution of (1.1)-(1.4), (1.5a,b). Assume that for some $0<R_{0}<R, q^{0}(r)>0$ and $q^{1}(r) \geqq 0$ for $R_{0}<r<R$. Also assume that $S^{0}(x) \geqq S_{0}$ for all $x \in \mathbf{R}^{2}$. Then $T$ is necessarily finite. Moreover, in the case when $\gamma=2$ the local existence time $T$ is bounded from above by $(c / \sigma)\left[\rho_{0} / B_{0}\right]^{2}$ as $B_{0} \rightarrow 0$, where $B_{0}=\frac{1}{2} \int_{R_{0}}^{R} q^{0}(\lambda) d \lambda$.

The idea of the proof of Theorem 2, as in the three-dimensional case, is motivated by previous blow-up results for nonlinear wave equations in twoand three-space dimensions [7], [8], [10].

\section{Proof of Theorem 2}

For simplicity, we first consider the case $\gamma=2$. Let $(\rho, u, S) \in C^{1}\left(\mathbf{R}^{2} \times\right.$ $[0, T))$ be a solution of $(1.1)-(1.4),(1.5 \mathrm{a}, \mathrm{b})$. Let $\omega(x, r)=\left(x_{1}-r\right)^{2}$ and define

$$
Q(r, t)=\int_{x_{1}>r} \omega(x, r)\left(\rho(x, t)-\rho_{0}\right) d x .
$$

Then by Lemma $1, Q(r, t)=0$ for $r \geqq R+\sigma t, t \geqq 0$ and

$$
Q(r, t)=\int_{r}^{\sigma t+R} \omega(x, r) \int_{-\left[(\sigma t+R)^{2}-x_{1}^{2}\right]^{1 / 2}}^{\left[(\sigma t+R)^{2}-x_{1}^{2}\right]^{1 / 2}}\left(\rho(x, t)-\rho_{0}\right) d x_{2} d x_{1} .
$$

Now, by using equation (1.1) and integration by parts it follows that

$$
\begin{aligned}
\frac{\partial}{\partial t} Q(r, t) & =\int_{x_{1}>r} \omega(x, r) \rho_{t}(x, t) d x=-\int_{x_{1}>r} \omega(x, r) \nabla \cdot(\rho u) d x \\
& =\int_{x_{1}>r} \nabla \omega(x, r) \cdot \rho u d x .
\end{aligned}
$$

In (2.2), we have used the fact that $u=0$ for $|x| \geqq \sigma t+R$, and $\omega(x, r)=0$ on $x_{1}=r$. We conclude from (2.2) that $Q(r, t)$ is $C^{2}$ in $t$. Thus, by differentiating again and using (1.1), (1.2), we find that

$$
\begin{aligned}
\frac{\partial^{2}}{\partial t^{2}} Q(r, t) & =\int_{x_{1}>r} \nabla \omega(x, r) \cdot\left(\rho_{t} u+\rho u_{t}\right) d x \\
& =-\int_{x_{1}>r} \nabla \omega(x, r) \cdot[u \nabla \cdot(\rho u)+\rho(u \cdot \nabla) u+\nabla p] d x
\end{aligned}
$$


Since $\nabla p=\nabla\left(p-p_{0}\right)$ where $p_{0}=p\left(\rho_{0}, S_{0}\right)$ and $\nabla \omega(x, r)=\left(2\left(x_{1}-r\right), 0\right)$, which vanishes on $x_{1}=r$, then integration by parts yields

$$
\begin{aligned}
\frac{\partial^{2}}{\partial t^{2}} Q(r, t)= & \int_{x_{1}>r} \Delta \omega(x, r)\left(p-p_{0}\right) d x \\
& +\int_{x_{1}>r} \rho\left[u_{1}^{2} \omega_{x_{1} x_{1}}+2 u_{1} u_{2} \omega_{x_{1} x_{2}}+u_{2}^{2} \omega_{x_{2} x_{2}}\right] d x \\
= & \int_{x_{1}>r} 2\left(p-p_{0}\right) d x+\int_{x_{1}>r} 2 \rho u_{1}^{2} d x \\
\geqq & \int_{x_{1}>r}\left(\frac{\partial}{\partial r}\right)^{2} \omega(x, r)\left(p-p_{0}\right) d x .
\end{aligned}
$$

Since $\omega(x, r)=\partial / \partial r(\omega(x, r))=0$ on $x_{1}=r$, we then have

Thus,

$$
\left(\frac{\partial}{\partial r}\right)^{2} Q(r, t) \geqq\left(\frac{\partial}{\partial r}\right)^{2} \int_{x_{1}>r} \omega(x, r)\left(p-p_{0}\right) d x .
$$

$$
\begin{aligned}
{\left[\left(\frac{\partial}{\partial t}\right)^{2}-\sigma^{2}\left(\frac{\partial}{\partial r}\right)^{2}\right] Q(r, t) } & \geqq\left(\frac{\partial}{\partial r}\right)^{2} \int_{x_{1}>r} \omega(x, r)\left[p-p_{0}-\sigma^{2}\left(\rho-\rho_{0}\right)\right] d x \\
& \equiv\left(\frac{\partial}{\partial r}\right)^{2} G(r, t) \equiv \widetilde{G}(r, t),
\end{aligned}
$$

where

$$
G(r, t)=\int_{x_{1}>r} \omega(x, r)\left[p-p_{0}-\sigma^{2}\left(\rho-\rho_{0}\right)\right] d x
$$

and

$$
\tilde{G}(r, t)=2 \int_{x_{1}>r}\left[p-p_{0}-\sigma^{2}\left(\rho-\rho_{0}\right)\right] d x .
$$

Note that if $\gamma=2$ then $\sigma^{2}=2 A \rho_{0} e^{S_{0}}$. Also, as long as $u$ is $C^{1}$, the particle paths

$$
\frac{d x}{d t}=u(x, t), \quad x(0, \xi)=\xi
$$

exist and equation (1.3) implies that $S(x, t)$ is constant along these paths. Since (by assumption) $S^{0}(x) \geqq S_{0}$, then $S(x, t) \geqq S_{0}$. Thus, $p(\rho, S) \geqq p\left(\rho, S_{0}\right)$. Consequently,

$$
\begin{aligned}
p-p_{0}-\sigma^{2}\left(\rho-\rho_{0}\right) & \geqq A e^{S_{0}}\left[\rho^{2}-\rho_{0}^{2}-2 \rho_{0}\left(\rho-\rho_{0}\right)\right] \\
& =\frac{\sigma^{2}}{2 \rho_{0}}\left(\rho-\rho_{0}\right)^{2},
\end{aligned}
$$

which leads to the lower bounds

$$
G(r, t) \geqq \frac{\sigma^{2}}{2 \rho_{0}} \int_{x_{1}>r} \omega(x, r)\left(\rho-\rho_{0}\right)^{2} d x \geqq 0,
$$


and

$$
\widetilde{G}(r, t) \geqq \frac{\sigma^{2}}{\rho_{0}} \int_{x_{1}>r}\left(\rho-\rho_{0}\right)^{2} d x \geqq 0 .
$$

Inversion of the one-dimensional d'Alembertian $\square_{\sigma}=(\partial / \partial t)^{2}-\sigma^{2}(\partial / \partial r)^{2}$ in (2.3) gives

$$
Q(r, t) \geqq Q^{0}(r, t)+\frac{1}{2 \sigma} \int_{0}^{t} \int_{r-\sigma(t-\tau)}^{r+\sigma(t-\tau)} \widetilde{G}(\lambda, \tau) d \lambda d \tau
$$

where

$$
Q^{0}(r, t)=\frac{1}{2}\left[q^{0}(r+\sigma t)+q^{0}(r-\sigma t)\right]+\frac{1}{2 \sigma} \int_{r-\sigma t}^{r+\sigma t} q^{1}(\lambda) d \lambda .
$$

Now, define the $C^{2}$-function

$$
F(t)=\int_{0}^{t}(t-\tau) \int_{\sigma \tau+R_{0}}^{\sigma \tau+R} r^{-\alpha} Q(r, \tau) d r d \tau,
$$

where $\frac{3}{4}<\alpha<1$ which will be fixed throughout. Our goal is to obtain a differential inequality for $F(t)$ which shows that $F(t)$ cannot be $C^{2}$ on $[0, \infty)$, and hence the theorem is proved.

Direct computation shows that

$$
\begin{aligned}
F^{\prime \prime}(t) & =\int_{\sigma t+R_{0}}^{\sigma t+R} r^{-\alpha} Q(r, t) d r \\
& \geqq \int_{\sigma t+R_{0}}^{\sigma t+R} r^{-\alpha} Q^{0}(r, t) d r+\frac{1}{2 \sigma} \int_{\sigma t+R_{0}}^{\sigma t+R} r^{-\alpha} \int_{0}^{t} \int_{r-\sigma(t-\tau)}^{r+\sigma(t-\tau)} \widetilde{G}(\lambda, \tau) d \lambda d \tau d r \\
& \equiv I_{1}(t)+I_{2}(t),
\end{aligned}
$$

respectively. The assumptions $q^{0}(r)>0$ and $q^{1}(r) \geqq 0$ for $R_{0}<r<R$, yield that

$$
\begin{aligned}
I_{1}(t) & \geqq \frac{1}{2} \int_{\sigma t+R_{0}}^{\sigma t+R} r^{-\alpha} q^{0}(r-\sigma t) d r \\
& \geqq \frac{1}{2}(\sigma t+R)^{-\alpha} \int_{R_{0}}^{R} q^{0}(\lambda) d \lambda \equiv B_{0}(\sigma t+R)^{-\alpha},
\end{aligned}
$$

where $B_{0}=\frac{1}{2} \int_{R_{0}}^{R} q^{0}(\lambda) d \lambda>0$. In order to estimate $I_{2}(t)$ from below, we write

$$
\begin{aligned}
I_{2}(t)= & \frac{1}{2 \sigma} \int_{0}^{t-R_{1}} \int_{\sigma \tau+R_{0}}^{\sigma \tau+R} \tilde{G}(\lambda, \tau) \int_{\sigma t+R_{0}}^{\lambda+\sigma(t-\tau)} r^{-\alpha} d r d \lambda d \tau \\
& +\frac{1}{2 \sigma} \int_{t-R_{1}}^{t} \int_{\sigma \tau+R_{0}}^{\sigma \sigma t-\sigma \tau+R_{0}} \tilde{G}(\lambda, \tau) \int_{\sigma t+R_{0}}^{\lambda+\sigma(t-\tau)} r^{-\alpha} d r d \lambda d \tau \\
& +\frac{1}{2 \sigma} \int_{t-R_{1}}^{t} \int_{2 \sigma t-\sigma \tau+R_{0}}^{\sigma \tau+R} \tilde{G}(\lambda, \tau) \int_{\lambda-\sigma(t-\tau)}^{\lambda+\sigma(t-\tau)} r^{-\alpha} d r d \lambda d \tau \\
\equiv & I_{2}^{1}(t)+I_{2}^{2}(t)+I_{2}^{3}(t),
\end{aligned}
$$


respectively, where $R_{1}=\left(R-R_{0}\right) / 2 \sigma$. In $I_{2}^{1}$ we have

$$
\begin{aligned}
\int_{\sigma t+R_{0}}^{\lambda+\sigma(t-\tau)} r^{-\alpha} d r & \geqq(\lambda+\sigma(t-\tau))^{-\alpha}\left(\lambda-\sigma \tau-R_{0}\right) \\
& \geqq c(\sigma t+R)^{-\alpha} t^{-1}(t-\tau)\left(\lambda-\sigma \tau-R_{0}\right)^{2} \\
& \geqq c \sigma(\sigma t+R)^{-\alpha-1}(t-\tau)\left(\lambda-\sigma \tau-R_{0}\right)^{2}
\end{aligned}
$$

in $I_{2}^{2}$ we have

$$
\begin{aligned}
\int_{\sigma t+R_{0}}^{\lambda+\sigma(t-\tau)} r^{-\alpha} d r & \geqq(\sigma t+R)^{-\alpha}\left(\lambda-\sigma \tau-R_{0}\right) \\
& \geqq c \sigma(\sigma t+R)^{-\alpha-1}(t-\tau)\left(\lambda-\sigma \tau-R_{0}\right)^{2}
\end{aligned}
$$

and in $I_{2}^{3}$ we have

$$
\begin{aligned}
\int_{\lambda-\sigma(t-\tau)}^{\lambda+\sigma(t-\tau)} r^{-\alpha} d r & \geqq 2 \sigma(\sigma t+R)^{-\alpha}(t-\tau) \\
& \geqq c \sigma(\sigma t+R)^{-\alpha-1}(t-\tau)\left(\lambda-\sigma \tau-R_{0}\right)^{2}
\end{aligned}
$$

By using the estimates $(2.12 \mathrm{a}-\mathrm{c})$, it follows that

$$
I_{2}(t) \geqq c(\sigma t+R)^{-\alpha-1} \int_{0}^{t}(t-\tau) \int_{\sigma \tau+R_{0}}^{\sigma \tau+R}\left(\lambda-\sigma \tau-R_{0}\right)^{2}\left(\frac{\partial}{\partial \lambda}\right)^{2} G(\lambda, \tau) d \lambda d \tau .
$$

Now, by integration by parts and using the fact that $G(\lambda, \tau)=$ $\partial / \partial \lambda(G(\lambda, \tau))=0$ for $\lambda=\sigma \tau+R$, one finds

$$
\begin{aligned}
I_{2}(t) & \geqq c(\sigma t+R)^{-\alpha-1} \int_{0}^{t}(t-\tau) \int_{\sigma \tau+R_{0}}^{\sigma \tau+R} G(\lambda, \tau) d \lambda d \tau \\
& \geqq c \frac{\sigma^{2}}{\rho_{0}}(\sigma t+R)^{-\alpha-1} \int_{0}^{t} \int_{\sigma \tau+R_{0}}^{\sigma \tau+R} \int_{x_{1}>\lambda}(t-\tau) \omega(x, \lambda)\left(\rho(x, \tau)-\rho_{0}\right)^{2} d x d \lambda d \tau \\
& \equiv c \frac{\sigma^{2}}{\rho_{0}}(\sigma t+R)^{-\alpha-1} I_{3}(t) .
\end{aligned}
$$

However, Schwarz's inequality yields

(2.13a) $F(t)^{2} \leqq I_{3}(t) \int_{0}^{t}(t-\tau) \int_{\sigma \tau+R_{0}}^{\sigma \tau+R} \lambda^{-2 \alpha} \int_{x_{1}>\lambda,|x|<\sigma \tau+R} \omega(x, \lambda) d x d \lambda d \tau$.

Thus,

$$
I_{2}(t) \geqq c \frac{\sigma^{2}}{\rho_{0}}(\sigma t+R)^{-\alpha-1}(J(t))^{-1} F(t)^{2}
$$

where

$$
J(t)=\int_{0}^{t}(t-\tau) \int_{\sigma \tau+R_{0}}^{\sigma \tau+R} \lambda^{-2 \alpha} \int_{x_{1}>\lambda,|x|<\sigma \tau+R} \omega(x, \lambda) d x d \lambda d \tau
$$


We estimate $J(t)$ as follows:

$(2.15)$

$$
\begin{aligned}
J(t) & =2 \int_{0}^{t}(t-\tau) \int_{\sigma \tau+R_{0}}^{\sigma \tau+R} \lambda^{-2 \alpha} \int_{\lambda}^{\sigma \tau+R}\left(x_{1}-\lambda\right)^{2}\left[(\sigma \tau+R)^{2}-x_{1}^{2}\right]^{1 / 2} d x_{1} d \lambda d \tau \\
& \leqq c \int_{0}^{t}(t-\tau) \int_{\sigma \tau+R_{0}}^{\sigma \tau+R} \lambda^{-2 \alpha}\left[(\sigma \tau+R)^{2}-\lambda^{2}\right]^{1 / 2}(\sigma \tau-\lambda+R)^{3} d \lambda d \tau \\
& \leqq c \int_{0}^{t}(t-\tau)(\sigma \tau+R)^{-2 \alpha+1 / 2} d \tau \\
& \leqq \frac{c}{\sigma^{2}}(\sigma t+R),
\end{aligned}
$$

since $\frac{3}{4}<\alpha<1$. Thus, (2.14) and (2.15) yield

$$
I_{2}(t) \geqq c \frac{\sigma^{4}}{\rho_{0}}(\sigma t+R)^{-\alpha-2} F(t)^{2}, \quad t \geqq 0 .
$$

Now, the combination of (2.9), (2.10) and (2.16) yields the inequalities

$$
\begin{array}{lll}
F^{\prime \prime}(t) \geqq B_{0}(\sigma t+R)^{-\alpha}, & t \geqq 0, \\
F^{\prime \prime}(t) \geqq c \frac{\sigma^{4}}{\rho_{0}}(\sigma t+R)^{-\alpha-2} F(t)^{2}, & & t \geqq 0 .
\end{array}
$$

We now proceed to show that $F(t)$ has a finite life span. First note that, since $F(0)=F^{\prime}(0)=0$, then $F^{\prime \prime}(t) \geqq 0, F^{\prime}(t) \geqq 0$ and $F(t) \geqq 0$ for all $t \geqq 0$. After integrating $(2.17 \mathrm{a})$ twice one finds that

$$
F(t) \geqq c \frac{B_{0}}{\sigma^{2}}(\sigma t+R)^{-\alpha+2}, \quad t \geqq k_{0} \equiv \frac{31 R}{\sigma} .
$$

Now, using (2.18) and interpolation with (2.17b) one has

$$
F^{\prime \prime}(t) \geqq c \frac{B_{0} \sigma^{2}}{\rho_{0}}(\sigma t+R)^{-2 \alpha} F(t),
$$

for $t \geqq k_{0} \equiv 31 R / \sigma$. Multiply (2.19) by $F^{\prime}(t)$ and integrate from $k_{1} \geqq k_{0}$ to $t$; one finds

$$
\begin{aligned}
F^{\prime}(t)^{2} & \geqq F^{\prime}\left(k_{1}\right)^{2}+c \frac{B_{0} \sigma^{2}}{\rho_{0}} \int_{k_{1}}^{t}(\sigma \tau+R)^{-2 \alpha} \frac{d}{d \tau}\left\{F(\tau)^{2}\right\} d \tau \\
& \geqq F^{\prime}\left(k_{1}\right)^{2}+\mu(t) F(t)^{2}-\mu\left(k_{1}\right) F\left(k_{1}\right)^{2},
\end{aligned}
$$

where $\mu(t)=c\left(B_{0} \sigma^{2} / \rho_{0}\right)(\sigma t+R)^{-2 \alpha}$. Since $F(0)=0$ and $F^{\prime \prime}(t) \geqq 0$, the mean value theorem yields that $F\left(k_{1}\right) \leqq k_{1} F^{\prime}\left(k_{1}\right)$. Choose $k_{1} \geqq k_{0}$ and sufficiently large so that $k_{1}^{2} \mu\left(k_{1}\right) \geqq 1$. Since $\frac{3}{4}<\alpha<1$, we can choose $k_{1} \equiv \max \left\{1, k_{0},(c / \sigma)\left(\rho_{0} / B_{0}\right)^{1 /(2-2 \alpha)}\right\}$. It now follows from (2.20) that

$$
\begin{aligned}
F^{\prime}(t)^{2} & \geqq F^{\prime}\left(k_{1}\right)^{2}+\left(k_{1}^{2} \mu\left(k_{1}\right)\right)^{-1}\left[\mu(t) F(t)^{2}-\mu\left(k_{1}\right) F\left(k_{1}\right)^{2}\right] \\
& \geqq\left(k_{1}^{2} \mu\left(k_{1}\right)\right)^{-1} \mu(t) F(t)^{2},
\end{aligned}
$$


or

$$
F^{\prime}>\frac{1}{k_{1}}\left(\sigma k_{1}+R\right)^{\alpha}(\sigma t+R)^{-\alpha} F(t), \quad t \geqq k_{1} .
$$

It follows fre at

$$
\left.\frac{F(t)}{F\left(k_{1}\right)}\right] \geqq \frac{\left(\sigma k_{1}\right)^{\alpha-1}}{1-\alpha}(\sigma t+R)^{1-\alpha},
$$

for $t \geqq 2 k_{1}$

$$
F(t) \quad\left[(1-\alpha)^{-1}\left(\sigma k_{1}\right)^{\alpha-1}(\sigma t+R)^{1-\alpha}\right], \quad t \geqq 2 k_{1} .
$$

It is easy to vt

$$
\exp \left[(1-\alpha)^{-1}\left(\sigma k_{1}\right)^{\alpha-1}(\sigma t+R)^{1-\alpha}\right] \geqq(\sigma t+R)^{2 \alpha+4},
$$

for $t \geqq 3 k_{1}$. So,

$$
F(t) \geqq F\left(k_{1}\right)(\sigma t+R)^{2 \alpha+4}, \quad t \geqq 3 k_{1} .
$$

Now, using (2.22) and interpolating with $(2.17 \mathrm{~b})$, we have

$$
\begin{aligned}
F^{\prime \prime}(t) & \geqq c \frac{\sigma^{4}}{\rho_{0}}(\sigma t+R)^{-\alpha-2} F(t)^{1 / 2} F(t)^{3 / 2} \\
& \geqq c \frac{\sigma^{4}}{\rho_{0}} F\left(k_{1}\right)^{1 / 2} F(t)^{3 / 2}
\end{aligned}
$$

for $t \geqq 3 k_{1}$. Multiply (2.23) by $F^{\prime}(t)$ and then integrate from $3 k_{1}$ to $t$; we have

$$
F^{\prime}(t)^{2} \geqq A_{1}\left[F(t)^{5 / 2}-F\left(3 k_{1}\right)^{5 / 2}\right], \quad t \geqq 3 k_{1},
$$

where $A_{1}=c\left(\sigma^{4} / \rho_{0}\right) F\left(k_{1}\right)^{1 / 2}$. By the mean value theorem,

$$
F^{\prime}\left(3 k_{1}\right) \leqq \frac{F(t)-F\left(3 k_{1}\right)}{t-3 k_{1}} \leqq F^{\prime}(t), \quad t \geqq 3 k_{1},
$$

and $F\left(3 k_{1}\right) \leqq 3 k_{1} F^{\prime}\left(3 k_{1}\right)$. Thus,

$$
\begin{aligned}
F(t) & \geqq F\left(3 k_{1}\right)+\left(t-3 k_{1}\right) F^{\prime}\left(3 k_{1}\right) \\
& \geqq F\left(3 k_{1}\right)+\frac{\left(t-3 k_{1}\right)}{3 k_{1}} F\left(3 k_{1}\right) \\
& =\frac{t}{3 k_{1}} F\left(3 k_{1}\right) \\
& \geqq \frac{8}{3} F\left(3 k_{1}\right)
\end{aligned}
$$

for $t \geqq 8 k_{1}$. Therefore, it follows from (2.24)

$$
F^{\prime}(t)^{2} \geqq A^{2} F(t)^{5 / 2}, \quad t \geqq 8 k_{1},
$$


or

$$
F^{\prime}(t) \geqq A F(t)^{5 / 4}, \quad t \geqq 8 k_{1},
$$

where $A=c \sigma^{2} \rho_{0}^{-1 / 2} F\left(k_{1}\right)^{1 / 4}$. If the local existence time $T \leqq 10 k_{1}$, then it follows from the definition of $k_{1}$ that $T \leqq(c / \sigma)\left[\rho_{0} / B_{0}\right]^{1 / 2(1-\alpha)}$, as $B_{0} \rightarrow 0$. Since $\alpha \in\left(\frac{3}{4}, 1\right)$ is arbitrary, then we have

$$
T \leqq \frac{c}{\sigma}\left[\frac{\rho_{0}}{B_{0}}\right]^{2} \quad \text { as } B_{0} \rightarrow 0 .
$$

On the other hand, if the local existence time $T>10 k_{1}$, then a final integration of (2.25) from $8 k_{1}$ to $T$ yields that

$$
F\left(8 k_{1}\right)^{-1 / 4} \geqq c A T \text {. }
$$

Now, as $B_{0} \rightarrow 0$ then $k_{1}=(c / \sigma)\left[\rho_{0} / B_{0}\right]^{1 / 2(1-\alpha)}, \alpha \in\left(\frac{3}{4}, 1\right)$. So, it follows from (2.18) that

$$
F\left(k_{1}\right) \geqq \frac{c}{\sigma^{2}} \rho_{0}^{(2-\alpha) / 2(1-\alpha)} B_{0}^{-\alpha / 2(1-\alpha)} .
$$

Thus, (2.28) and (2.22) yield that

$$
F\left(8 k_{1}\right) \geqq \frac{c}{\sigma^{2}} \rho_{0}^{(\alpha+6) / 2(1-\alpha)} B_{0}^{-(3 \alpha+4) / 2(1-\alpha)} .
$$

Now, by using (2.27), (2.29), and the assumption that $T>10 k_{1}$, one easily obtains the inequality

$$
c\left[\frac{\rho_{0}}{B_{0}}\right]^{(\alpha+2) / 2(1-\alpha)} \leqq 1 .
$$

As $B_{0} \rightarrow 0$ and $\rho_{0}$ is fixed, (2.30) is impossible. Thus, as $B_{0} \rightarrow 0$ we must have $T \leqq 10 k_{1}$. Hence $(2.26)$ is valid.

We finally turn our attention to the general case, $\gamma>1$. In this case, the change occurs in $(2.4 \mathrm{c})$ and instead we have

$$
\begin{aligned}
p-p_{0}-\sigma^{2}\left(\rho-\rho_{0}\right) & \geqq A e^{S_{0}}\left[\rho^{\gamma}-\rho_{0}^{\gamma}-\gamma \rho_{0}^{\gamma-1}\left(\rho-\rho_{0}\right)\right] \\
& \equiv A e^{S_{0}} \psi\left(\rho, \rho_{0}\right) .
\end{aligned}
$$

Since $\rho^{\gamma}$ is convex, then

$$
\psi\left(\rho, \rho_{0}\right)=\rho^{\gamma}-\rho_{0}^{\gamma}-\gamma \rho_{0}^{\gamma-1}\left(\rho-\rho_{0}\right)>0
$$

for $\rho \neq \rho_{0}$. However, by Taylor's theorem one has

$$
\psi\left(\rho, \rho_{0}\right) \geqq C_{0}\left(\gamma, \rho_{0}\right)\left(\rho-\rho_{0}\right)^{2} \quad \text { for } 0<\rho<2 \rho_{0},
$$

and some positive constant $C_{0}\left(\gamma, \rho_{0}\right)$. On the other hand, there exists a positive constant $C_{1}(\gamma)$ such that

$$
\psi\left(\rho, \rho_{0}\right) \geqq C_{1}(\gamma)\left(\rho-\rho_{0}\right)^{\gamma} \quad \text { for } \rho \geqq 2 \rho_{0} .
$$


Therefore, there exists a positive constant $C\left(\gamma, \rho_{0}\right)$ such that

$$
\psi\left(\rho, \rho_{0}\right) \geqq C\left(\gamma, \rho_{0}\right) \Phi_{\gamma}\left(\rho-\rho_{0}\right),
$$

where $\Phi_{\gamma}$ is a nonnegative convex function given by

$$
\Phi_{\gamma}\left(\rho-\rho_{0}\right)= \begin{cases}\left(\rho-\rho_{0}\right)^{2}, & 0<\rho<2 \rho_{0}, \\ \left(\rho-\rho_{0}\right)^{\gamma}, & \rho \geqq 2 \rho_{0} .\end{cases}
$$

Finally, Jensen's inequality should be used instead of Schwarz's inequality in (2.13a). The rest of the details should then follow accordingly, and the resulting differential inequality still has a finite life span. However, the upper bound for the local existence time $T$ will be different from the one which we have obtained for the case $\gamma=2$.

\section{REFERENCES}

1. R. Courant and D. Hilbert, Methods of mathematical physics, II, New York, Interscience, 1962.

2. F. John, Formation of singularities in one-dimensional nonlinear wave propagation, Commun. Pure Appl. Math. 27 (1974), 377-405.

3. _ Blow-up of radial solutions of $u_{t t}=c^{2}\left(u_{t}\right) \Delta u$ in three space dimensions, Mat. Aplic. Comp. 4 (1) (1985), 3-18.

4. T. Kato, The Cauchy problem for quasilinear symmetric systems, Arch. Rat. Mech. Anal. $\mathbf{5 8}$ (1975), 181-205.

5. S. Klainerman and A. Majda, Compressible and incompressible fluids, Commun. Pure Appl. Math. 35 (1982), 629-651.

6. T. P. Liu, The development of singularities in the nonlinear waves for quasi-linear hyperbolic partial differential equations, J. Differ. Equations 33 (1979), 92-111.

7. M. Rammaha, Finite-time blow-up for nonlinear wave equations in high dimensions, Commun. Partial Differ. Equations 12 (6) (1987), 677-700.

8. __ On the blowing up of solutions to nonlinear wave equations in two space dimensions, J. Reine Angew. Math. 391 (1988), 55-64.

9. T. Sideris, Formation of singularities of solutions to nonlinear hyperbolic equations, Arch. Ration. Mech. Anal. 86 (4) (1984), 369-381.

10. __ Global behavior of solutions to nonlinear wave equations in three space dimensions, Commun. Partial Differ. Equations 8 (12) (1983) 1291-1323.

11. _ Formation of singularities in three-dimensional compressible fluids, Commun. Math. Phys. 101 (1985), 475-485.

12. S. Ukai, The incompressible limit and the initial layer of the compressible Euler equation, $\mathbf{J}$. Math. Kyoto Univ. 26 (2) (1986), 323-331.

Department of Mathematics and Statistics, University of Nebraska at Lincoln, LiNCOLN, NeBRASKa 68588 